\title{
Development of Rescue Material Transport UAV (Unmanned Aerial Vehicle)
}

\author{
Daeil Jo, Yongjin Kwon* \\ Department of Industrial Engineering, College of Engineering, Ajou University, Suwon, South Korea \\ Email: ^yk73@ajou.ac.kr
}

How to cite this paper: Jo, D. and Kwon, Y. (2017) Development of Rescue Material Transport UAV (Unmanned Aerial Vehicle). World Journal of Engineering and Technology, 5, 720-729. https://doi.org/10.4236/wjet.2017.54060

Received: October 18, 2017

Accepted: November 13, 2017

Published: November 16, 2017

Copyright ( 2017 by authors and Scientific Research Publishing Inc. This work is licensed under the Creative Commons Attribution International License (CC BY 4.0).

http://creativecommons.org/licenses/by/4.0/

(c) (i) Open Access

\begin{abstract}
Recently, the market for drones is growing rapidly. Commercial UAVs (Unmanned Aerial Vehicles, or drones) are increasingly being used for various purposes, such as geographic survey, rescue missions, inspection of industrial facilities, traffic monitoring and delivery of cargos and goods. In particular, the drones have great potential for life-saving operations. A missing person, for example, can be rapidly and effectively searched using a drone in comparison with the conventional search operations. However, there is no commercially available rescue UAV until now. The motivation for this study is to design an unmanned aerial vehicle capable of vertical takeoff and landing, while containing a high power propellant apparatus in order to lift a heavy cargo that contains rescue materials (such as water, food, and medicine). We used the EDF (Electric Ducted Fan) technology as opposed to the conventional motor and prop combination. The EDF can produce the power about three times higher than the motor-prop combination. This became suitable for transportation of rescue goods, and can be widely used in rescue operations in natural environments. Based on these results, the UAV for rescue material transport capable of heavy vertical takeoff and landing is developed, including airframe, flight control computer and GCS (ground control station).
\end{abstract}

\section{Keywords}

High-Powered Propellant, Vertical Take-Off and Landing, Rescue Drone, GCS (Ground Control Station), FCC (Flight Control Computer)

\section{Introduction}

In recent years, the use of drones or UAVs (unmanned aerial vehicles) is increasingly getting popular around the world. Drones are utilized in aerial photography for both personal hobbies and commercial uses. In industrial sectors, drones 
are deployed in facility inspection, power line inspections, and monitoring of fire and flood purposes. In almost every imaginable part, the drones are being used [1] [2]. The rescue operation is in no exception. Due to its fast flying characteristics, which can cover a wide area in less time, drones are now being used by law enforcement agencies for search missing persons. The current rescue drones are typically equipped with thermal image cameras; hence it can detect the missing person from the surroundings, even though the missing person is not visually identifiable from the surrounding environment. However, there is no rescue drones that can carry the emergency materials, such as water, food, blanket, and medicine, and being capable of conducting rescue operations [3] [4] [5]. In order to do this, the drones must have a high power propellant technology that is different from the conventional motor and prop combination. Additionally, the rescue drone should be able to vertically takeoff and land, while carrying the rescue materials. Therefore, the motivation of this study is to develop a rescue drone that is capable of conducting a search and rescue operation with onboard carbo capacity. Through this development, we design and produce the actual airframe and cargo handling device, set up the firmware SW of flight control computer, and run a flight stability test to validate the safe flying characteristics. We will actually build a structural drone that is still in the concept phase and prepare a technology base so that a large number of IPs can be created in the future.

Currently, various hobby and surveillance drones are being developed and commercially available in Korea, but no high power drones are developed yet, with the high load carrying capacity. Most hobby drones cannot carry enough load, and even the large size drone can only carry less than 1 or 2 Kilograms. The biggest agricultural drones can carry about 10 to 15 Kilograms of payload, but they are too large for police rescue operations [6] [7]. In this context, the drone should be small enough to fit in the police car and easy to handle. At the same time, the drone should be generating enough lifting power, so that it can carry the rescue materials for the stranded or missing person. This will complement the shortcomings of the conventional UAV that is lacking the power capacity. As the demand for UAV increases in the future, it will lay the foundations for developing various types of UAVs (courier, transportation, delivery, lifeguard, etc.) that can meet the customer demands.

\section{Development of Software and Hardware}

\subsection{Motors and Propellers}

The drones that we want to make in this study basically have to deliver relief goods to people located in the middle of the building in case of fire and emergency situation in high-rise building. At this point, the most important point is that the drones must be close to the outer wall of the building to deliver relief supplies. In case of EDF (Electric Ducted Fan), it is possible to approach close to the building because the rotating prop is contained inside the turbine housing. 
However, when the conventional propeller motor is used, the drone is difficult to approach the building because the exposed propeller may hit the structure and the drone will fall from the sky. Ultimately, this difference is the biggest reason to use the EDF motors in developing the rescue drone [8] [9] [10]. There are also considerations when comparing EDF and propeller motors. It is whether or not you can protect the safety of your finger when you fly it. Due to the nature of the relief donor drone, the person must directly reach out and remove the relief item from the box in the lower part of the drone. At this time, according to different rotation method of EDF motor and propeller motor, the safety protection of the finger is different. In other words, EDF drone do not have a risk of injuring your fingers unless you intentionally put your fingers into the turbine housing. However, in propeller motors, the propeller itself is located outside the drones, so there is a risk that the fingers can be cut any time. This means that if a propeller motor is used, the drones used for the rescue may cause secondary damage. Because of this, we have chosen a high thrust EDF, instead of conventional moto and prop combination. Electronic Ducted-Fan type airplane itself consists of engine, propeller, fixed wing and flap. Theoretically, moving the fluid flow from the rotating body to the duct increases the thrust efficiency by $41 \%$. However, the weight of ducts to be increased in comparison with the propeller should also be considered. The aim of this study is to lift a total weight of about $10 \mathrm{~kg}$, which has four EDFs, each of which can maintain the duration of $50 \%$ of the maximum thrust at a weight of up to $18 \mathrm{~kg}$, as shown in Figure 1.

\subsection{Battery Selection}

The battery is a major factor in controlling the drones' flight time and output. The larger the capacity of the battery, the longer the flight time, and the higher the number of cells in a series connected battery. The selected EDF requires a higher voltage than the propeller and motor combination, so we select a battery with 6 - 8 cells. The battery has a high stability and uses a lithium ion polymer material. This is because the electrolyte of the lithium ion battery is a liquid and basically has a problem in stability. We also considered trade-offs in battery capacity and weight. Electric vehicles' biggest dilemma is the proportional relationship between the capacity and weight. When a heavy battery is used in a

\begin{tabular}{cc} 
Dr. Mad Thrust $90 \mathrm{~mm} 12$ Blade Alloy EDF $1250 \mathrm{kv}$ \\
Weight & $615 \mathrm{~g}$ \\
MAX RPM & 37000 \\
MAX Power (10 sec.) Watt & 3700 \\
Continuous Power Watt & 3300 \\
Current & $112 \mathrm{~A}$ \\
Max Voltage & $30 \mathrm{~V}(8 \mathrm{~s})$ \\
\hline
\end{tabular}

Figure 1. Selected EDF: Highly safe, theoretically efficient EDF around the wing with Duct. Maximum thrust per EDF $4.5 \mathrm{~kg} \times 4=18 \mathrm{~kg}$ Expectations, excluding frame and self weight-Estimated thrust at least $8 \mathrm{~kg} / 900 \mathrm{~g} \times 4=3.6 \mathrm{~kg}$ (left), EDF Specification (right). 
EDF with a duct, the weight of an automatic cardioverter defibrillator (AED), a fire mask, a lantern, and other rescue materials need to be reduced. Also, the weight of the drone itself can be increased significantly. The selected battery is PT-B10000-NSR35 model and has specifications of $22.2 \mathrm{~V}, 6 \mathrm{~S} 1 \mathrm{P}, 35 \mathrm{C}+$, as shown in Figure 2. To meet the selected EDF output and minimum duration, the total capacitance that can be supplied by connecting two batteries in parallel was increased.

\subsection{Frame Design}

Four EDFs are designed in four directions to balance the drones. Fixtures for installing ESC (electric speed controller), battery holder, GPS transceiver and other fixtures are installed on the center top plate. In addition, a fixture for installing a structural goods transport box is installed in the lower part, and two supports for stably loading and unloading the drones are installed. The material of the frame was made of carbon material to minimize the weight of the drone, and some aluminum parts were also used, as shown in Figure 3. A cylindrical EDF fixture using a 3D printer was designed as an EDF dedicated frame for fixing each EDF. In the end, thanks to the frame made of carbon material, it was able to create a frame that can withstand a high-output EDF, while lightening the weight as much as possible.

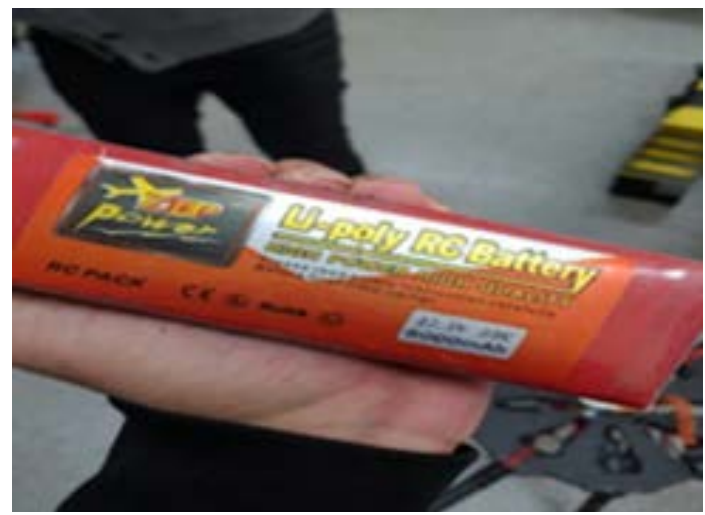

Figure 2. Selected batteries: Selecting a battery that can supply the appropriate voltage for the EDF output $(400 \mathrm{~g} \times 2=800 \mathrm{~g})$.
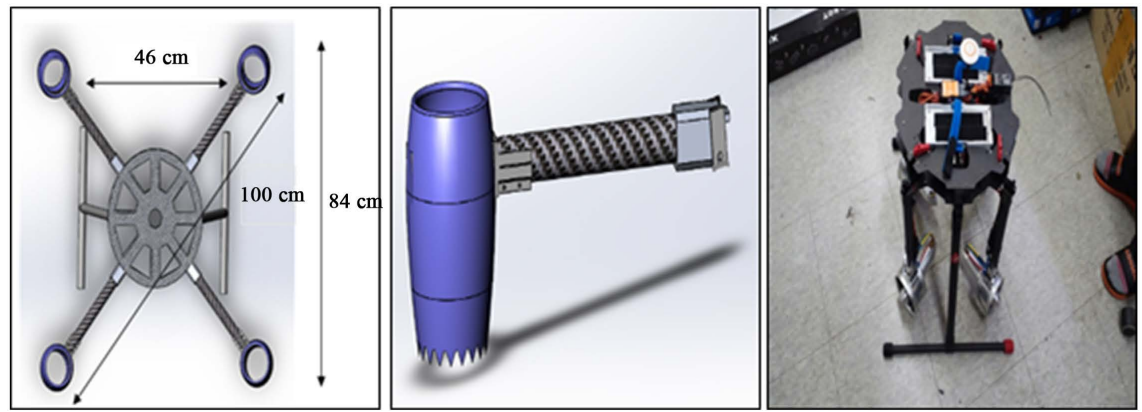

Figure 3. Frame drawing and specification: Carbon frame selection/2.2 kg (left), EDF fixture design drawing (center), Drone assembly and fabrication process (right). 


\subsection{Design and Manufacture of Rescue Material Box}

In order to minimize the imbalance in the flight of the drones, a rectangular box-shaped model penetrating the bottom of the drones was adopted. The vertical length is the minimum length, which is larger than the length of the frame including the EDF to avoid interference with the EDF. The height is defined as the maximum length that can be installed between the support and center of the drones frame. In order to deliver the rescue supplies, the drones must be approached through the windows and the being rescued person must reach the arm and bring the rescue supplies. Since the drones are not fully accessible to the building, the length of the arm of the person should be considered with the minimum length. In this case, it is necessary to design the arm length so that it is not difficult for both a person with a shorter arm and a person with a longer arm to approach, as shown in Figure 4. The design should be based on the average (498 mm) of the arm length first decile (lowest quartile) for women between 10 and 80 years of age. Height, width, and length are based on the minimum and maximum length.

The material called "Foam-max" was considered because of the weight and the strength of the box itself. Foam-max is foamed and compressed by using PVC as raw material and it is very easy to process while maintaining the advantages of plastic and wood. It was to create boxes and to make room for speakers in the middle. Speakers installed in the drones are able to communicate with the stranded person who are in need. It is also possible to reserve extra batteries in case of remaining space. In addition, a partition was installed to prevent internal objects from moving during the flight, thus preventing the center of gravity of the drones from being disturbed [11] [12].

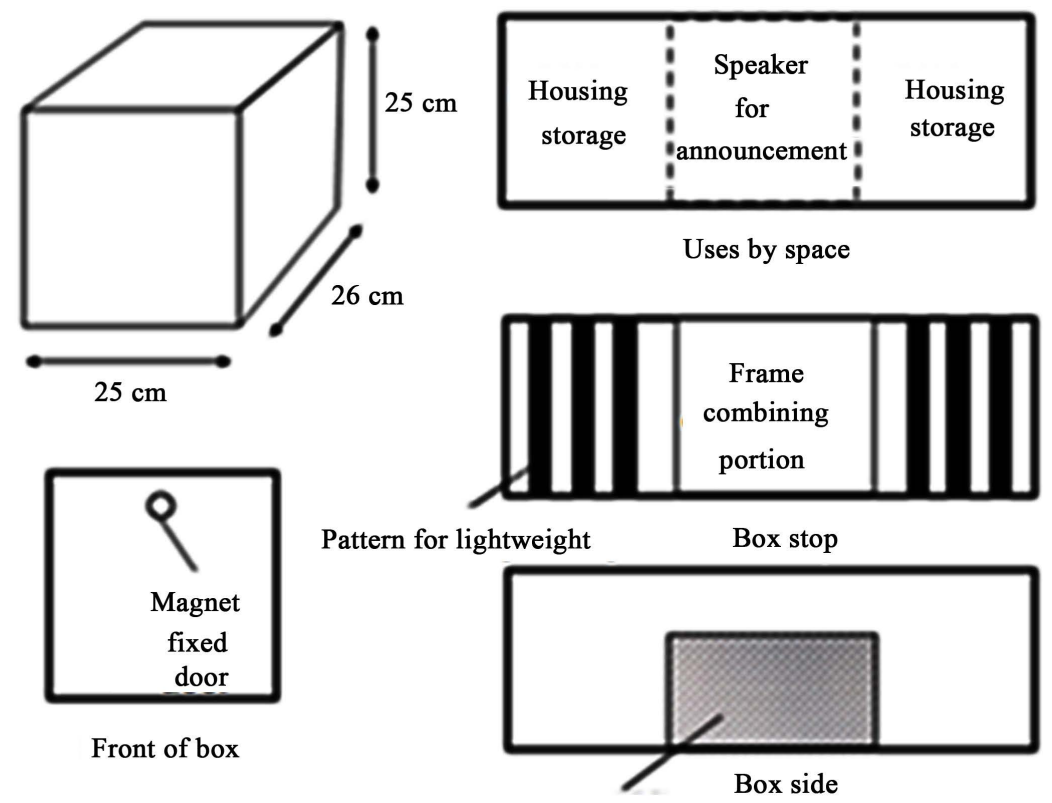

Perforated network for speaker transmission

Figure 4. Prototype of rescue material box. 


\subsection{Type of Rescue Material and Delivery Method}

In the case of storage space for rescue materials, it is possible to store about $1 \mathrm{ki}$ logram of materials. It is possible to store items according to the purpose and to greatly increase the survival rate in case of fire, such as oxygen cans, masks, firefighting clothes, fire extinguisher, water, water towel, and so on. Structural rope, radio, and emergency medicine can also be stored. In addition, if a cardiac arrest patient is present, the attached box can be removed and the cardiac defibrillator, portable AED, attached to the lower part of the drone can be delivered. When a high-rise building is delivered to a rescue target in the middle or upper floors, it approaches the building window and draws the rope connected to the storage bag connected to the storage box to deliver the rescue goods. As illustrated in Figure 5, the operation procedure is as follows:

1) Pull out the line outside the box.

2) At the same time the door is opened, the rescue materials come out.

3) Hold the rescue supplies.

4) Pull the rescue supplies.

\section{Research and Development Results}

The details of the developed drone is given in Figure 6. The drone was tested and evaluated for its flight characteristics. Figure 7 shows the test flight of takeoff and landing. The test was proceeded smoothly and the flight was very stable.

In this study, EDF was selected because of the safety problem of the propeller, but it was not able to produce $4.5 \mathrm{~kg}$ of thrust as indicated in the product specification. $4.5 \mathrm{~kg}$ When 4 EDFs were used, the specification gave $18 \mathrm{~kg}$ of thrust.
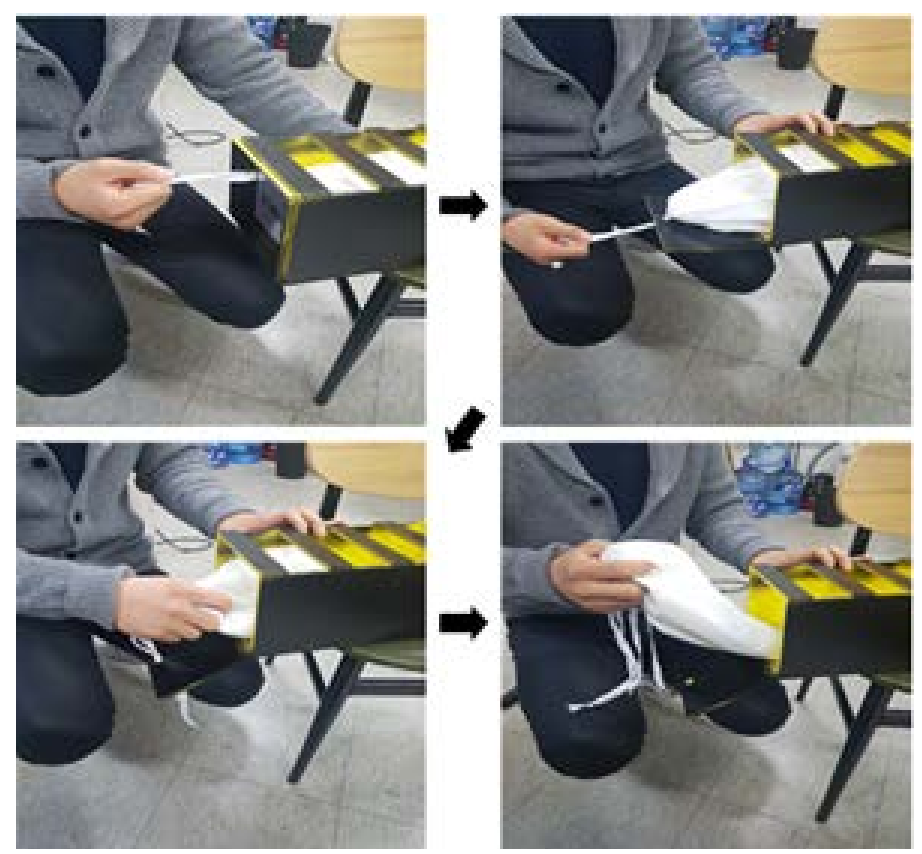

Figure 5. Rescue material transport system. 


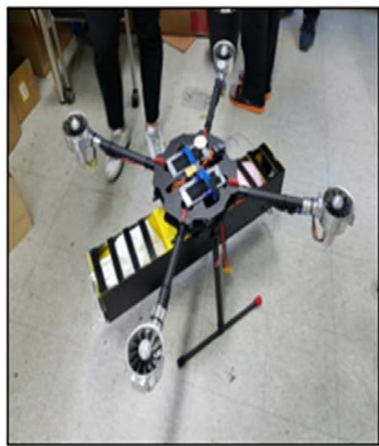

\begin{tabular}{|cc}
\hline \multicolumn{2}{c}{ EDF } \\
\hline Duration (Per change) & 3 minutes \\
\hline Noise & $74 \mathrm{~dB}$ \\
Weight & $3140 \mathrm{ollars}$ \\
Cost & $8.3 \mathrm{~kg}$ \\
Speed & $1.4 \mathrm{~m} / \mathrm{s}$ \\
Actual loading weight & $1.2 \mathrm{~kg}$ \\
Theoretical maximum thrust & $18 \mathrm{~kg}$ \\
\hline Average thrust & $9.5 \mathrm{~kg}$ \\
\hline
\end{tabular}

Figure 6. Developed drone with structural box and relief items and its specification.

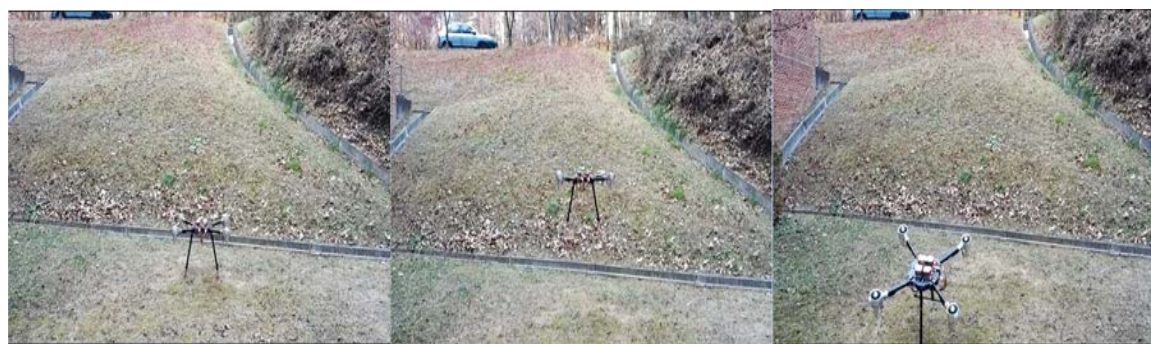

Figure 7. Drones test flight: takeoff and landing.

Except for the weight of the body, $10 \mathrm{~kg}$ of thrust was expected, but the actual thrust was only $10.5 \mathrm{~kg}$. And a run-time of $1 / 10$ th compared to propeller aircraft capable of flying over 30 minutes.

Thrust determination equation is as follows [9] [10] [11] [12].

$$
\text { Power }=\text { Prop Const } * r^{\text {Power Factor }}
$$

The equation to determine the propeller was as follows.

$$
T=\frac{\pi}{4} D^{2} \rho v \Delta v
$$

$T=$ thrust $[\mathrm{N}]$

$D=$ propeller diameter $[\mathrm{m}]$

$v=$ velocity of air at the propeller $[\mathrm{m} / \mathrm{s}]$

$\Delta v=$ velocity of air accelerated by propeller $[\mathrm{m} / \mathrm{s}]$

$\rho=$ density of air $\left[1.225 \mathrm{~kg} / \mathrm{m}^{3}\right]$

The thrust ratio was calculated by excluding the air velocity (v) related factor through the thrust formula. And when using a propeller, it was calculated that four times as much thrust was possible. This is because the diameter of the propeller has the greatest influence on thrust. To develop an EDF that produces the same thrust as a $4 \times$ long propeller, it required 16 times the revolutions per second, which drastically reduced battery life. In addition, it required a high voltage of $30 \mathrm{~V}$ compared to a propeller requiring $22.2 \mathrm{~V}$. In the case of a commercially available battery, the maximum voltage was $6 \mathrm{~s}(3.7 \mathrm{~V} \times 4=22.2 \mathrm{~V})$. Finally, the weight of the EDF itself was $900 \mathrm{~g}$, totaling $3.6 \mathrm{~kg}$. It was about 9 times the weight of the propeller and I think it was the biggest weight gain factor 
in the airframe design that we are trying to reduce 1g. In order to compare the exact thrust between the propeller and the EDF, I think it would be necessary to consider vortex elimination and air compression rise through the duct. In order to actually introduce EDF, which has the advantage of safety, we need three things: 1, lighter weight, 2. efficiency, and 3. propeller size [13] [14] [15] [16]. Figures 8-12 shows the flight characteristics data, all of which shows a very stable flight characteristics.

\begin{tabular}{ccccc}
\hline & Wingspan & Rotational Speed/s & T/pv \\
\hline Prop & $45.72 \mathrm{~cm}$ & 133.20 & 26.77 \\
EDF & $11 \mathrm{~cm}$ & 616.67 & 6.64 \\
\hline & Required & Used Battery & Thrust & Weight \\
& Voltage & Voltage & Ratio & \\
\hline Prop & $22.2 \mathrm{v}(6 \mathrm{~s})$ & $22.2(6 \mathrm{~s})$ & 4 & $220(\mathrm{~g})$ \\
\hline EDF & $30 \mathrm{v}(8 \mathrm{~s})$ & $22.2(6 \mathrm{~s})$ & 1 & $900(\mathrm{~g})$ \\
\hline
\end{tabular}

Figure 8. EDF and propeller comparison.

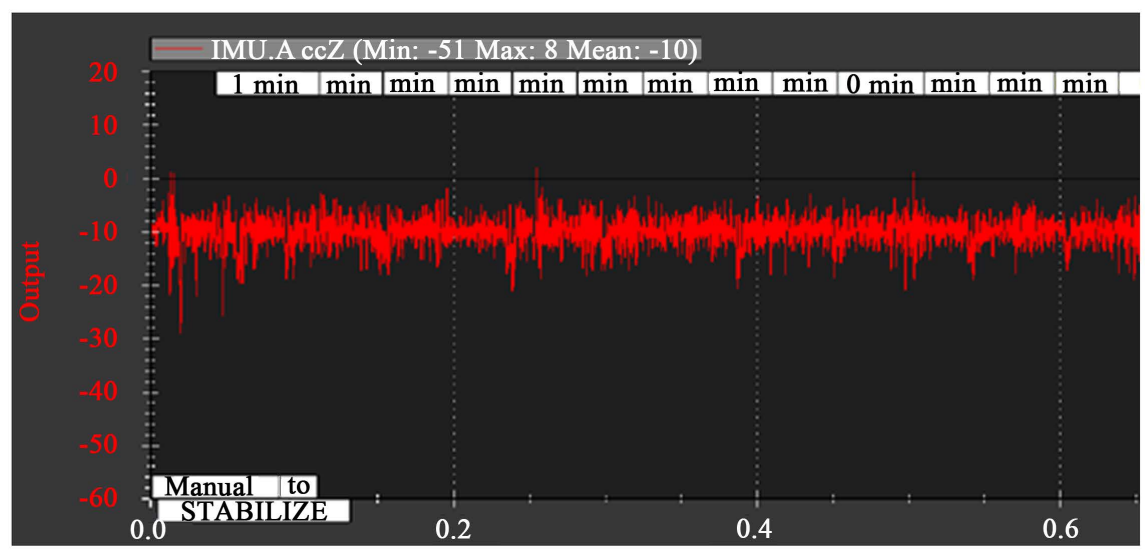

Figure 9. Z-axis acceleration data.

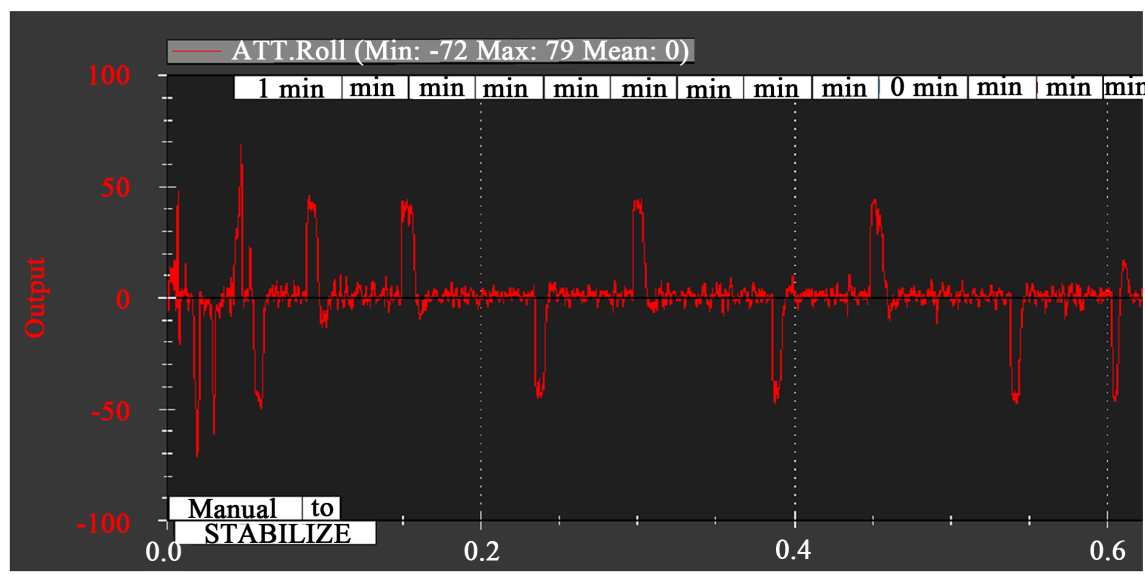

Figure 10. Bank angle data. 


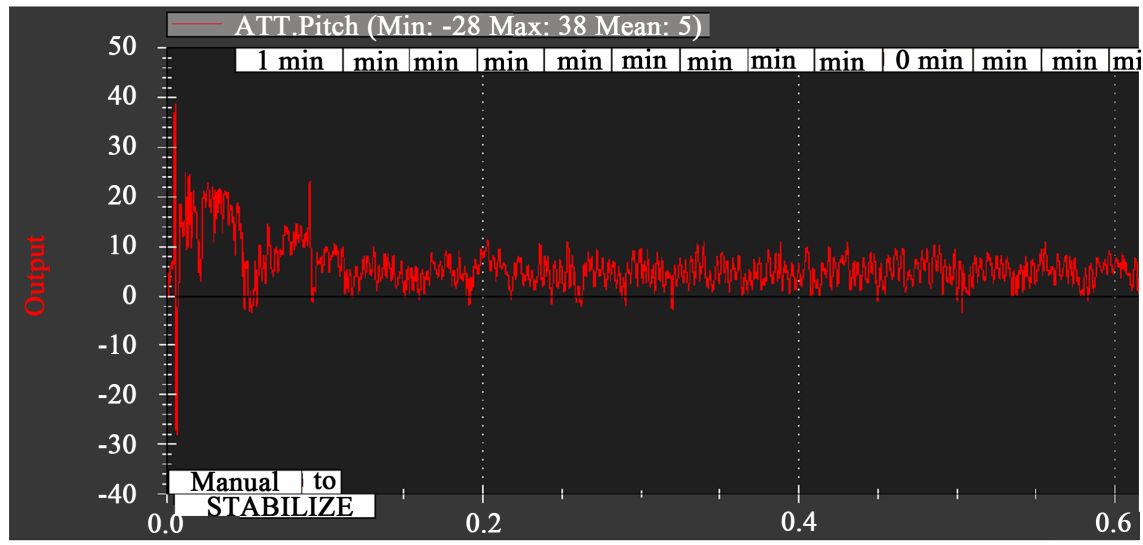

Figure 11. Pitch angle data.

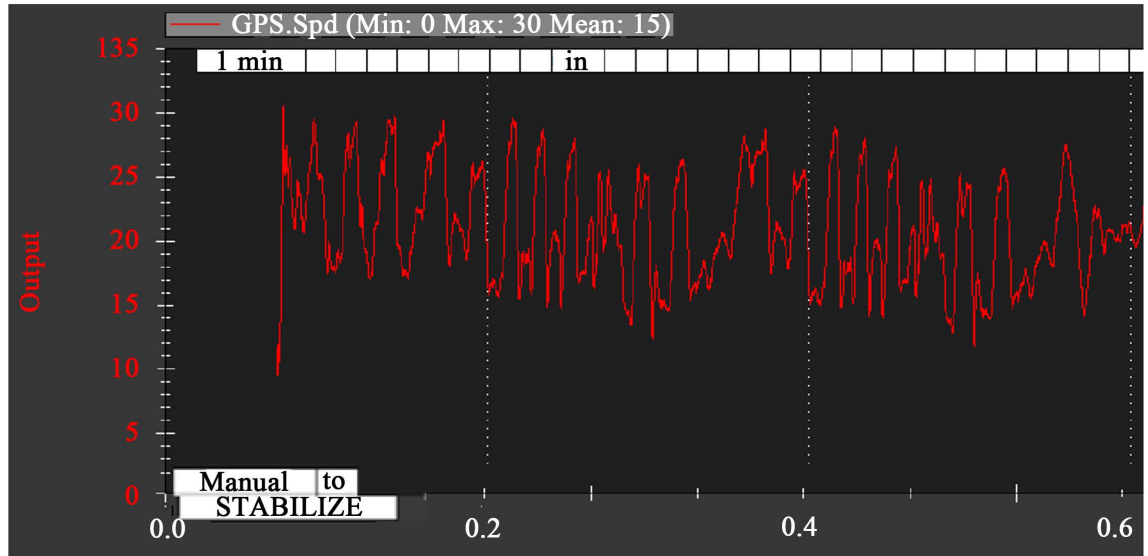

Figure 12. Speed data.

\section{Conclusion}

UAVs capable of high-power, vertical takeoff and landing can complement the disadvantages of conventional UAV's lack of power and maximize utilization throughout the industry. Through this technology development, we localize the core technology of UAV that can control high power and vertical flight stably. As the demand for UAV increases in the future, it will lay the foundations for developing various types of UAVs (courier, transportation, delivery, lifeguard, etc.) that meet customer demand. Expected effects of meeting domestic demand, import substitution and export will be created. In the future, we will complement the problems analyzed in this study and design a new type of drone. In the end, we will develop more than $25 \mathrm{~kg}$ payload so that it can be utilized for life-saving and transportation of rescue materials.

\section{Acknowledgements}

This work was supported by the Ajou University research fund.

\section{References}

[1] Yu, S. and Kwon, Y. (2017) Development of VTOL Drone for Stable Transit Flight. 
Journal of Computer and Communications, 5, 36-43. https://doi.org/10.4236/jcc.2017.57004

[2] Yu, S. and Kwon, Y. (2017) Development of Multi-Purpose, Variable, Light Aircraft Simulator. Journal of Computer and Communications, 5, 44-52. https://doi.org/10.4236/jcc.2017.57005

[3] Jo, D. and Kwon, Y. (2017) Analysis of VTOL UAV Propellant Technology. Journal of Computer and Communications, 5, 76-82. https://doi.org/10.4236/jcc.2017.57008

[4] De la Torre, G.G., Ramallo, M.A. and Cervantes, E.G. (2016) Workload Perception in Drone Flight Training Simulators. Computers in Human Behavior, 64, 449-454.

[5] Liu, Z. and Li, H. (2006) Research on Visual Objective Test Method of High-Level Flight Simulator. Xitong Fangzhen Xuebao (Journal of System Simulation).

[6] Luan, L.-N. (2013) Augmenting Low-Fidelity Flight Simulation Training Devices via Amplified Head Rotations. Loughborough University.

[7] Lee, S. and Lim, K. (2008) PCB Design Guide Book. Sehwa Publishing Co., South Korea.

[8] Kang, M. and Shin, K. (2011) Electronic Circuit. Hanbit Media, South Korea.

[9] Kim, D., Kim, J. and Yoon, S. (2016) Development and Validation of Manned and Unmanned Aircraft Simulation Engine for Integrated Operation in NAS. Journal of the Korean Society for Aeronautical \& Space Sciences, 44, 423-430.

[10] Sponholz, C.-B. (2015) Conception and Development of a Universal, Cost-Efficient and Unmanned Rescue Aerial Vehicle. Techische Hochschule Wildau Technical University of Applied Sciences.

[11] Guerrero, M.E., Mercado, D.A. and Lozano, R. (2015) IDA-PBC Methodology for a Quadrotor UAV Transporting a Cable-Suspended Payload. 2015 International Conference on Unmanned Aircraft Systems (ICUAS), Denver, CO, 9-12 June 2015, 470-476. https://doi.org/10.1109/ICUAS.2015.7152325

[12] Gomez, C. and Purdie, H. (2016) UAV-Based Photogrammetry and Geocomputing for Hazards and Disaster Risk Monitoring-A Review. Geoenvironmental Disasters, 3, 1. https://doi.org/10.1186/s40677-016-0060-y

[13] Lee, E., Kim, S. and Kwon, Y. (2016) Analysis of Interface and Screen for Ground Control System. Journal of Computer and Communications, 4, 61-66. https://doi.org/10.4236/jcc.2016.45009

[14] Kwon, Y., Heo, J., Jeong, S., Yu, S. and Kim, S. (2016) Analysis of Design Directions for Ground Control Station (GCS). Journal of Computer and Communications, 4, 1-7. https://doi.org/10.4236/jcc.2016.415001

[15] Yu, S., Heo, J., Jeong, S. and Kwon, Y. (2016) Technical Analysis of VTOL UAV. Journal of Computer and Communications, 4, 92-97. https://doi.org/10.4236/jcc.2016.415008

[16] Hong, J., Baek, S., Jung, H., Kim, S. and Kwon, Y. (2015) Usability Analysis of Touch Screen for Ground Operators. Journal of Computer and Communications, 3, 133-139. https://doi.org/10.4236/jcc.2015.311021 